\title{
Sken\&agraphie
}

SKIINagraphII Coulisses des arts du spectacle et des scènes

émergentes

$5 \mid 2018$

Juste la fin du monde, de Lagarce à Dolan

\section{Jean-Luc Lagarce et l'écriture scénaristique : Entretien avec Gérard Bouysse, coscénariste du scénario Des Mensonges}

Propos recueillis par Alexis Leprince

\section{Alexis Leprince}

\section{(2) OpenEdition}

\section{Journals}

Édition électronique

URL : http://journals.openedition.org/skenegraphie/1537

DOI : 10.4000/skenegraphie.1537

ISSN : 2553-1875

Éditeur

Presses universitaires de Franche-Comté

Édition imprimée

Date de publication : 1 janvier 2018

Pagination : 179-190

ISBN : 978-2-84867-5609-8

ISSN : $1150-594 \mathrm{X}$

Référence électronique

Alexis Leprince, « Jean-Luc Lagarce et l'écriture scénaristique : Entretien avec Gérard Bouysse,

coscénariste du scénario Des Mensonges », Sken\&agraphie [En ligne], 5 | 2018, mis en ligne le 01 janvier 2019, consulté le 02 mai 2019. URL : http://journals.openedition.org/skenegraphie/1537 ; DOI :

10.4000/skenegraphie.1537

Ce document a été généré automatiquement le 2 mai 2019.

Presses universitaires de Franche-Comté 


\title{
Jean-Luc Lagarce et l'écriture scénaristique : Entretien avec Gérard Bouysse, coscénariste du scénario Des Mensonges
}

\author{
Propos recueillis par Alexis Leprince
}

\section{Alexis Leprince}

S'il est un grand cinéphile - son Journal en témoigne à chaque page, Jean-Luc Lagarce n'est pas qu'un spectateur assidu : il aspire aussi à être un praticien. Au fil des années, il s'investit dans des projets cinématographiques: ainsi, dans les années 1980, il écrit quelques critiques pour Libération, sous le pseudonyme de Paul Dasthré. Il travaille ensuite à un scénario, Quai des Grands-Augustins, adapté du roman de Jean Rhys, mais ce scénario restera sans suite. À la fin des années 80, il commence, avec la complicité du Centre international de création vidéo (CICV) de Montbéliard, le tournage de son film vidéo, Journal 1 (voir l'entretien avec François Berreur, Catherine Derosier-Pouchous et Patrick Zanoli). En même temps qu'il finit de tourner et commence à monter son film, il rencontre Gérard Bouysse, avec lequel il se met à écrire un scénario, nommé dans ses archives Des Mensonges. Gérard Bouysse a bien voulu évoquer avec nous l'origine de ce projet et le processus d'écriture de ce scénario coécrit avec Jean-Luc Lagarce. On en trouvera quelques extraits choisis dans le "Carnet de la création ".

TOUT D'ABORD, PEUX-TU NOUS DIRE COMMENT TU AS RENCONTRÉ JEAN-LUC LAgARCE ET COMMENT EST VENUE L'IDÉE DE TRAVAILLER ENSEMBLE À L'ÉCRITURE d'UN SCÉNARIO ?

Je l'ai connu par l'intermédiaire d'un ami comédien, Olivier Achard. J'ai rencontré pour la première fois Jean-Luc Lagarce lorsque je suis venu voir Music-hall, pièce dans laquelle Olivier jouait. Nous avons rapidement sympathisé, et dès le début il m'avait confié son envie de cinéma. Il avait déjà fait une adaptation, à partir d'un texte de Jean Rhys $^{1}$ il me semble, mais il n'en était pas content, et il avait toujours ce fort désir de cinéma.

Après Music-hall, on devient donc assez proches, on se voit pratiquement toutes les semaines, autour d'un petit groupe d'une dizaine de personnes qui se constitue avec 
notamment Olivier, mais aussi Lorraine Groleau, l'assistante de Jacques Rivette, et Pierre Novion, chef opérateur. Pour Jean-Luc, je crois qu'il était agréable de se retrouver dans un milieu différent que le milieu théâtral qu'il fréquentait habituellement.

Ensuite, très rapidement, on décide de travailler ensemble. Se pose alors le problème du sujet, même si pour nous, le sujet était un peu secondaire : nous étions plus fascinés par Tarkovski que par Claude Sautet, même si nous avions beaucoup d'estime pour le travail de Sautet. Dans mes cartons, j'avais accumulé des notes sur cette histoire, devenue commune aujourd'hui, de la rencontre du monde théâtral et du monde des grands médias, tel que TF1, qui sont alors en pleine constitution. On est au début de la privatisation des chaînes de télévision et du star-système avec les animateurs.

Je propose ces notes à Jean-Luc, et on décide de partir là-dessus. Ce qui nous intéresse, c'est l'affrontement de ces deux blocs et, en même temps, à la fois la fascination qu'exercent les médias sur le théâtre, et la situation complexe de ces nouveaux médias qui cherchent une forme de validation dans l'ancien territoire intellectuel artistique. Les deux blocs s'attirent mais ils ne peuvent pas se mélanger. Ces notes, au début, s'appellent Les Menteurs, car les deux blocs mentent tous les deux. C'est ce qui a posé problème à la commission ${ }^{2}$. Il y a eu des oppositions farouches. Certains détestaient l'image du théâtre que l'on donnait à voir ; par contre d'autres, comme Christine Pascal ou Bernard Verlay nous ont tout aussi farouchement défendus.

\section{LA RÉALISATION dU fiLm A DONC ÉTÉ STOPPÉE PAR LE REFUS DE LA COMMISSION ?}

C'est surtout la disparition de Jean-Luc qui a stoppé la réalisation du film. Malgré le refus de la commission, un producteur, Humbert Balzan, était intéressé par le film et on avait commencé à se rapprocher de lui. On devait présenter à nouveau le projet devant une commission présidée par Jérôme Deschamps cette fois-ci, mais Jean-Luc est mort, et comme je traversais moi-même quelques problèmes, je n'avais plus le temps de travailler dessus. Plus tard, j'ai trouvé que le sujet avait trop vieilli. Lorsqu'on a écrit le scénario, le sujet n'avait pas été traité, mais quelques années plus tard, un certain nombre de films s'étaient emparés de la thématique, dont Masques de Claude Chabrol, par exemple. L'actualité du film disparaissait, Jean-Luc n'était plus là, cela s'est perdu.

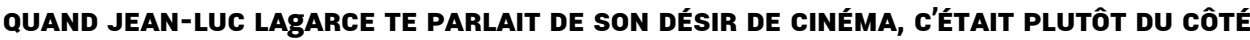
DE L'ÉCRITURE SCÉNARISTIQUE, OU AUSSI DU CÔTÉ DE LA RÉALISATION ?

Au départ, c'était l'écriture, mais j'ai toujours pensé qu'il y avait un désir de réalisation derrière, je l'ai toujours vécu comme ça. Sur ce projet, j'étais en réalisation, et Jean-Luc Lagarce était en coscénariste, mais je crois que l'idée de Jean-Luc Lagarce, c'était d'être présent tout au long du processus, parce que je pense qu'il avait un désir de réalisation fort. C'est un désir qu'il a toujours eu, mais il y avait des obstacles techniques importants, surtout à l'époque.

Par rapport à cette question de l'écriture, il faut aussi bien comprendre que le texte qui reste, c'est vraiment un texte en cours. On était plus préoccupé par Tarkovski que par Sautet, mais quand on lit le scénario, on pense plus à Sautet qu'à Tarkovski! Notre préoccupation c'était de trouver un producteur. À l'époque où l'on commence à écrire, on est encore dans le vieux système du cinéma : il faut d'abord avoir un scénario qui est validé par des producteurs, des acteurs. Mais l'intention c'est d'en faire autre chose au tournage, comme pour une mise en scène : le même texte, selon que c'est Bob Wilson, Tadeusz Kantor ou Pina Bausch qui s'en empare, cela ne donne pas la même chose. Voilà pourquoi je n'ai pas voulu qu'on publie le scénario : c'est quelque chose qui est 
écrit pour monter le projet. C'est un rapport à l'écriture qui est donc particulier, car l'écriture se termine et s'achève pendant le tournage, puis avec le montage...

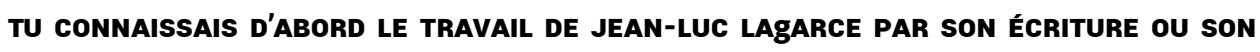
TRAVAIL DE MISE EN SCÈNE ?

Plutôt par la mise en scène. Mais j'ai bien sûr été frappé par l'écriture. À l'époque, on ne pouvait pas rater la qualité de l'écriture quand on voyait une pièce de Jean-Luc Lagarce. J'ai un souvenir très particulier de Music-hall, car il y a une réussite à tous niveaux qui était absolue. La mise en scène lui ressemblait beaucoup, avec des systèmes très chorégraphiés, une grande importance donnée au placement des personnages, à leurs mouvements. Dans Music-hall, on sentait ce travail de chorégraphie qui s'exprimait d'ailleurs très bien dans le texte quand il est dit que la jeune fille entrait par le fond: ce type d'indications me semble très caractéristique des mises en scène au millimètre de Jean-Luc. Je me souviens également de sa mise en scène du Malade imaginaire, avec un circuit, des passages obligés; de même dans La Cantatrice chauve. Il y avait un réglage qui était plus de l'ordre de la chorégraphie, ce qui le différencie d'olivier Py par exemple, chez qui les acteurs sont déchaînés sur le plateau, avec des mouvements de groupe qui rappellent Strehler. Jean-Luc Lagarce était dans quelque chose de très réglé, très chorégraphié, plus proche de Pina Bausch que de Strehler.

\section{COMMENT A-T-IL REÇU LE FAIT QUE LE SCÉNARIO AIT ÉTÉ REFUSÉ ?}

C'était un coup dur, car c'était beaucoup de travail. Il fallait relativiser car, comme je l'ai dit, un producteur était intéressé : ce n'était pas un coup d'arrêt mais juste un ralentissement. Mais Jean-Luc Lagarce était dans une urgence, il avait le sentiment qu'il fallait qu'il travaille, qu'il avance, qu'il fasse des choses, car il y avait ce risque qu'il disparaisse.

Un MOt maintenant sUR le processus de travall entre Vous deUX. Dans les ARCHIVES DE JEAN-LUC LAgARCE, LES VERSIONS DU SCÉNARIO QUE L'ON A SONT ACCOMPAgNÉES d'ENVELOPPES ET DE LETTRES DE TA PART, CE QUI LAISSE IMAgINER UN TRAVAIL À DISTANCE ET BEAUCOUP D'ALLER-RETOURS ENTRE VOUS. TU M'AS DIT Également que vous vous retrouviez pour des séances de travall aU sélect. COMMENT S'EST DÉROULÉ LE PROCESSUS d'ÉCRITURE ENTRE VOUS?

Au début, je lui envoie donc l'assemblage de notes, qui est surtout un assemblage de personnages, Les Menteurs. Ensuite, on a commencé à écrire au fil de l'eau : on écrivait des scènes entières que l'on se soumettait l'un à l'autre, que chacun réécrivait, et ainsi de suite. On écrivait les premières versions d'abord chacun de notre côté car on n'était pas capables d'écrire l'un et l'autre ensemble, mais par contre nous avons des discussions sur ce qu'on a écrit. Cela exigeait une grande intimité entre nous, sinon ce type de processus ne fonctionne pas, d'autant que l'essentiel du film, pour nous, ce n'est pas tant les moments où les gens parlent ou se parlent mais les moments où ils ressentent les choses, les moments où chaque personnage est seul. On discutait beaucoup de cela. On était conscient que ce serait un problème pour la réalisation. Pour nous, cela structurait le film: les grandes solitudes de chacun, le fait aussi que chacun est seul dans un groupe.

\section{VOUS VOUS ÊTES ATTACHÉS TOUS LES DEUX À DES SCÈNES EN PARTICULIER, OU VOUS AVEZ} TOUT ÉCRIT EN COMMUN ?

Les scènes, c'est-à-dire les situations, c'était presque systématiquement moi qui les écrivait, et après nous faisions le traitement de la situation ensemble. Jean-Luc me disait toujours qu'il ne savait écrire qu'une seule chose, l'histoire d'un homme qui 
revient chez lui. Il disait qu'il ne savait pas faire autre chose, que ce n'était pas intéressant, qu'il n'en finissait jamais de réécrire Par les villages. J'écrivais donc les scènes au départ, puis on faisait le traitement tous les deux, mais c'est réellement très poreux : à certains moments, il y a presque une ligne de lui, une ligne de moi et ainsi de suite. On se voyait au minimum une fois par semaine pour travailler. À un moment, il avait des injections à faire tous les jours, vers 9 heures du matin; donc, à certaines périodes, je l'appelais à 9 heures pendant le temps du traitement pour discuter du scénario. Écrire ensemble, c'est une intimité joyeuse, sinon cela ne peut pas marcher.

LE SCÉNARIO S'EST TITRÉ LES MENTEURS, OU RETOUR À L'AUTOMNE, ET IL EST INSCRIT DANS LES ARCHIVES SOUS LE TITRE DES MENSONgES. EST-CE QUE CE CHANgEMENT DE TITRE EST SYMPTOMATIQUE d'UN PROCESSUS d'ÉCRITURE HACHÉ, AVEC DE gRANDS CHANgEMENTS AU COURS DU TRAVAIL, DE gRANDS BOULEVERSEMENTS DANS L'ORIENTATION DU PROJET ? OU AU CONTRAIRE EST-CE QUE L'ÉCRITURE A SUIVI UN CHEMIN PLUS CALME ET LINÉAIRE ?

C'était plutôt linéaire. Par exemple, on n'a jamais eu la moindre opposition avec JeanLuc, cela a toujours été extrêmement fluide dans le travail entre nous, et je pense que c'est lié au fait qu'on avait des parcours très semblables : nous partagions un certain isolement par rapport à la famille, pour des raisons très différentes; nous n'étions pas parisiens au départ, on avait un parcours professionnel différent mais on partageait les mêmes références littéraires, cinématographiques, théâtrales et artistiques en général. La seule opposition, la seule fois où on s'est vraiment engueulé, c'était à cause de la politique, dans le cadre d'un repas, car lui était très rocardien et moi quasiment anarchiste ! Mais ça n'était réellement qu'un détail.

AS-TU SENTI QUE SON EXPÉRIENCE DE DRAMATURgE, D'HOMME DE THÉÂTRE, INFLUENÇAIT SA MANIÈRE D'ÉCRIRE, LUI DONNAIT DES RÉFLEXES D'ÉCRITURE PARTICULIERS ?

Oui. Par exemple, dans la scène d'ouverture du scénario, il introduit une sorte de numéro music-hall, avec des hommes qui font les boys... On ressent qu'il voit déjà la mise en scène dans l'écriture de la scène. Après, ça disparaît un peu, on se concentre sur les personnages, il comprend qu'on n'écrit pas tout à fait de la même manière pour le cinéma et pour le théâtre. Au théâtre, quand on écrit le texte, il me semble qu'on est déjà plus ou moins dans la mise en scène, c'est déjà une projection sur la scène, tandis qu'au cinéma on n'envisage pas de la même manière les possibilités d'images. Il faut gérer le regard du spectateur plus qu'au théâtre.

Pour le dire brièvement, au théâtre, c'est une proposition dans une fenêtre, avec le cadre de scène, surtout dans le théâtre à l'italienne - dans le théâtre contemporain, maintenant, c'est différent, notamment avec l'arrivée de la vidéo. Rapidement, il comprend qu'au cinéma on n'est pas prisonniers du cadre de scène, qu'au cinéma on écrit une sorte de squelette pour ce qui se fera ensuite. C'est pour cela que je n'ai pas souhaité publier ce scénario, puisque c'est comme un texte auquel il manque les voyelles.

Il avait comme qualité d'être un formidable dialoguiste. Il fallait que les dialogues puissent résonner en bouche d'une manière juste, et il arrivait à ce résultat en jouant les dialogues pendant l'écriture. Il écrivait, jouait le texte, et réécrivait à partir de cela. Il hurlait presque le texte pour l'écrire. C'était très drôle, parce que moi je n'avais jamais pratiqué ou vu ça. Tous les deux, pendant nos séances, on lisait, presque hurlait le texte. Car ces textes étaient destinés non à être lus, mais à être dits et entendus. C'est quelque chose qu'on retrouvait dans son phrasé au quotidien : il avait un phrasé, une présence, une élocution qui fascinait et frappait tout de suite. 
TU AS SENTI QU'IL S'EST MIS PEU À PEU dANS LES CODES DE L'ÉCRITURE SCÉNARISTIQUe ?

C'est quelque chose dont on a beaucoup discuté, car un scénario n'est vraiment qu'un texte de base. Il fallait comprendre que l'écriture se ne finissait pas avec ce premier état du scénario, mais qu'il constituait un point d'appui, et que l'écriture se continue ensuite avec la caméra, puis avec le montage. C'est un processus plus long, et c'est pourquoi il faut s'attacher aux personnages plus qu'à la situation qui, une fois qu'elle est posée, est anecdotique. L'intérêt, ce sont les présences que l'on va pouvoir faire exister, le souci principal est de créer les conditions pour qu'elles puissent émerger. C'était vraiment une écriture qui porte en elle une vision future du travail, plus encore qu'au théâtre.

LE SCÉNARIO PRÉSENTE UN METTEUR EN SCÈNE QUI ACCEPTE UN PROJET AUQUEL IL CROIT Peu, dans le but de gagner beaucoup d'ARgent et de pouvoir, À L'AVenir, Être plus LIBRE DE CRÉER CE QU'IL SOUHAITE AVEC SA COMPAgNIE DE THÉÂTRE. JUSQUE DANS SON titre, le scénario ÉVOQUe Le thème de LA COMPromission ARTISTIQUe, de LA tricherie, Que l'on Retrouve dans les pièces de Jean-luc lagarce. Cette THÉMATIQUE EST APPARUE RAPIDEMENT ?

Oui, les premières notes s'appelaient «Des Menteurs ». C'était une espèce de liste de personnages structurée par une anaphore, qui revenait à chaque fin de présentation d'un personnage, «il ment» ou «il est menteur», je ne me souviens plus. C'était ça l'essentiel: d'une manière ou d'une autre, tout le monde ment, mais presque en étant sincère en même temps; c'est cela qui est intéressant. C'était vraiment la base de l'histoire, du projet.

et cette IDÉe QUe les personnages réécrivent Ce Qu'ONT dit leURS PRoches ? ON SAIT QUE LA QUESTION DE LA RÉÉCRITURE EST IMPORTANTE CHEZ LAgARCE...

C'est un classique de l'auteur. C'est classique mais c'est quelque chose qui est difficile à éviter. On s'était vraiment posé la question de savoir si on conservait cela alors que c'est une antienne, mais on trouvait que c'était tellement vrai que, bien que ce soit classique, on ne pouvait pas faire l'impasse dessus. Tous les auteurs se servent de leur entourage et c'est extrêmement désagréable pour les proches, puisque quelque chose qui a été dit dans une situation se retrouve dans un autre contexte ; le propos est tordu et, pour l'entourage, c'est toujours très désagréable. C'est un malaise qui existe autour des auteurs d'une manière à peu près systématique. Il me semble que cette idée n'avait pas de signification particulière pour nous, que c'était simplement un motif classique, puisque, pour les auteurs, c'est un problème qui se règle finalement assez rapidement ; sinon on ne s'en sort pas dans le travail d'écriture.

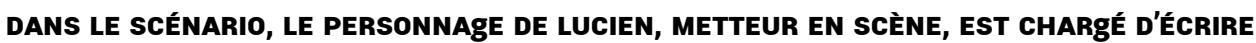
L'ADAPTATION D'UN TEXTE RETROUVÉ DE TCHEKHOV, MAIS IL NE PARLE PAS RUSSE, ET L'ON SE REND COMPTE PEU À PEU QU'IL ÉCRIT SON PROPRE TEXTE, SANS LE DIRE. ON SAIT QUE JEAN-LUC LAgARCE A ÉCRIT BEAUCOUP D'ADAPTATIONS, QUE PARFOIS AUSSI IL NE S'EST PAS SENTI RECONNU EN TANT Qu'AUTEUR. EST-Ce QUe Ce PERSONNAge de LUCIEN A quelque chose à voir aVec Jean-luc lagarce, plus particulièrement aVec sa POSITION D'AUTEUR, QU'IL AURAIT EU DU MAL À ASSUMER?

L'histoire de Lucien part de la proposition de Pelletier $^{3}$ : un texte de Tchekhov a été retrouvé, et on décide d'en faire un coup publicitaire. Le texte est sans doute nul, donc on demande à un auteur en vue, qui peut ressembler à Jean-Luc Lagarce, d'en faire quelque chose. Mais on est dans le mensonge des deux côtés, et plutôt qu'un auteur qui ne s'assume pas, il s'agit aussi pour Lucien de ce qui est tellement caractéristique de notre époque, cette réification des choses, cette transformation de tout en objet de profit: Tchekhov est dans le domaine public, mais si l'on modifie quelques lignes au 
texte de base, on en devient l'auteur...

Jean-Luc Lagarce avait parfois un sourire grinçant sur ces problèmes de droits, d'appropriation, mais, lui, il se positionnait totalement comme auteur, c'était très clair pour lui. Il n'avait aucun doute sur sa qualité, sur son statut d'auteur ; il était très lucide sur son niveau.

Après, quand L'Arche a refusé ses textes, il l'a mal vécu. C'était une humiliation. De même, sur le scénario, on a eu une lettre très insultante du Losange, où l'on nous a dit de changer de métier, qu'ils n'avaient jamais lu un truc aussi mal écrit. Mais encore une fois cela nous a fait plutôt rire. Quand L'Arche a refusé de publier ses pièces, c'était très dur, mais il avait quand même conscience de son talent ; il n'en doutait pas. À la fin, les deux-trois dernières années, une forme de reconnaissance est arrivée, mais la plupart du temps c'était quand même très difficile.

\section{JUSTEMENT, APRÈs JUSTE LA fin du MONDE, PENDANT CETTE PÉRIOdE OÙ VOUS TRAVAILlez

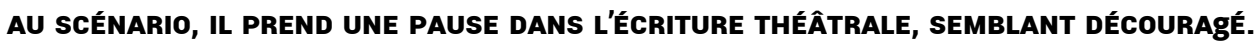 CE N'EST JAMAIS UN SENTIMENT QUI AFFLEURAIT DANS VOS CONVERSATIONS ?}

C'est une chose différente. Moi je parle de sa qualité d'auteur presque technique, et de ce point de vue, il sait qu'il est auteur. Là où il y a un doute qui intervient chez Jean-Luc Lagarce, c'est de savoir si ce qu'il écrit a une existence profonde. C'est dans ces moments-là qu'il disait écrire toujours la même chose, et cela le faisait un peu souffrir parce que, en plus, cela rejoignait ce qu'avait fait Handke dans Par les villages... C'est une inquiétude partagée par tous les artistes : est-ce que ce que je fais n'est que du savoirfaire ou est-ce que j'atteins à quelque chose ? Quand l'artiste a un doute sur ce qu'il fait, et qu'il a le sentiment que ce qu'il fait, il ne le fait que par savoir-faire, c'est très douloureux. En écriture théâtrale, Jean-Luc était confronté à cela.

Avec le scénario, c'était différent ; il n'y a pas la rapidité qu'on trouve dans l'écriture théâtrale. Le scénario c'est un processus long, et Jean-Luc n'aimait pas ça, par nature, à titre personnel, mais aussi parce qu'il est inquiet par rapport à la maladie. Il ressentait la difficulté pendant les dernières années de ne pas réussir à atteindre un état de création fluide, délivré du doute, alors qu'il l'avait vécu joyeusement avant. Dans les années 1990, il semblait à la recherche d'un second souffle.

\section{JEAN-LUC LAgARCE ÉtAIT FASCINÉ PAR L'ÉCRITURE D'HERVÉ GUIBERT, QUI A ÉCRIT UN SCÉNARIO AVEC PATRICE CHÉREAU, L'HOMME BLESSÉ. EST-CE QUE C'ÉTAIT UN MODÈLE QUI A PU gUIDER VOTRE TRAVAIL ? QUELLES AUTRES RÉFÉRENCES VOUS gUIDAIENT ?}

Non, nous n'avons pas évoqué le travail scénaristique de Guibert comme un modèle. Hervé Guibert, comme Jean-Luc Lagarce, était atteint du SIDA, et c'était forcément une préoccupation chez Jean-Luc. C'était aussi une thématique qui était très présente, avec un film comme Les Nuits fauves, plus tard Philadelphia. Avec Hervé Guibert, il y avait cette dimension commune non pas forcément d'un combat mais d'une compréhension de la situation de cette époque, qu'on a beaucoup oubliée aujourd'hui. Mais cela ne se retrouve pas dans le scénario. Jean-Luc n'était pas très attiré par l'autofiction, ses études de philosophie lui donnaient toujours une forme de distance par rapport au réel, et sa vie à lui ne rejoignait pas ce qu'il écrivait. Encore une fois, il avait par exemple d'excellentes relations avec sa famille!

Nos références étaient plutôt Godard, Tarkovski, et Pina Bausch. Il y avait Tchekhov qui était présent aussi. Pina Bausch, cela peut paraitre étonnant, mais son travail nous intéressait, notamment par rapport à l'aspect chorégraphique des mises en scène de Jean-Luc. C'est quelque chose sur lequel on se rejoignait, car j'ai une manière de 
tourner assez réglée. Il y a des scènes du scénario qui sont écrites pour être mises en scène avec une préoccupation liée à Pina Bausch, dans les déplacements de gens, les manières d'être à l'image.

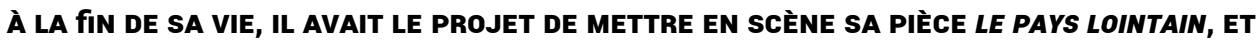
L'ON RETROUVE DANS SES ARCHIVES DE NOMBREUX DOCUMENTS SEMBLANT INDIQUER QU'IL SOUHAITAIT METTRE DE LA VIDÉO SUR SCÈNE. VOUS AVIEZ diALOgUÉ À CE PROPOS ?

Oui, et il avait déjà intégré de la vidéo au Théâtre de la Cité Internationale pour sa mise en scène d'Histoire d'amour, dans laquelle il y avait, si je me souviens bien, trois ou quatre écrans sur scène. Nous en avons beaucoup parlé car on était au tout début de la vidéo sur scène, on n'avait pas encore les grands écrans par exemple. On questionnait le sens que cela pouvait avoir, comment la vidéo et la théâtralité pouvaient se mélanger, travailler ensemble. À l'époque, la vidéo, c'était une image très contrastée, avec des couleurs criardes; on n'était pas dans une image type cinéma mais dans une image vidéo, donc cela créait des problèmes. Le mélange entre l'acteur et la vidéo était moins harmonieux qu'aujourd'hui. L'écran, c'était quelque chose de spécial au niveau de l'impact visuel et plastique. C'était difficile d'organiser l'harmonie entre le lieu théâtral et le lieu vidéo, car cette présence criarde sur les écrans cathodiques en 256 couleurs créait une violence.

On envisageait par exemple la vidéo comme un deuxième discours sur certains écrans, quelque chose de narratif ; cela pouvait être aussi un système de gros plans pour casser ce cadre de l'espace théâtral de l'époque, des gros plans qui du coup bouleverseraient la lecture, la distance, du spectateur par rapport à ce qui se passe sur scène. On réfléchissait, on envisageait les possibles, mais nous n'avons pas été au bout de la réflexion. On en était aux notes d'intuitions, au premier travail de défrichement, après il fallait faire des essais sur scène, mais Jean-Luc est mort avant.

Avant sa mort, nous avions également le projet de captation des pièces de Jean-Luc. Mais on ne voulait pas faire une captation classique : on voulait prendre un parti pris brechtien, souligner le fait que c'était joué, ne pas oublier qu'on était en train de faire une vidéo sur la pièce, ne pas du tout donner l'illusion qu'on est assis à l'orchestre quand on regarde la captation, garder cette distance. Cela posait tout un tas de questionnements qui étaient passionnants! Mais nous n'avons pas eu le temps de le faire.

\section{NOTES}

1. Il s'agit de Quai des Grands-Augustins, de Jean Rhys, dont Lagarce tirera un scénario.

2. La commission d'avance sur recettes avant réalisation.

3. Personnage de l'acteur-producteur dans le scénario, à la base du projet mais qui le quittera en cours pour prendre les rênes d'une émission de télévision. 
INDEX

Mots-clés : Jean-Luc Lagarce, Gérard Bouysse, Juste la fin du monde, Le Pays lointain, Des Mensonges, Music-Hall

\section{AUTEURS}

\section{ALEXIS LEPRINCE}

Alexis Leprince est doctorant boursier de la MSHE Claude Nicolas Ledoux à l'Université de Besançon Franche Comté où il poursuit une recherche en génétique sur l'œuvre théâtrale du dramaturge Jean-Luc Lagarce. Également praticien, il est le collaborateur régulier de la compagnie Artépo, en tant qu'assistant à la mise en scène et dramaturge. 